\title{
Convex Polygon Intersection Graphs
}

\author{
Erik Jan van Leeuwen ${ }^{1}$ and Jan van Leeuwen ${ }^{2}$ \\ 1 Department of Informatics, University of Bergen \\ P.O. Box 7803, N-5020 Bergen, Norway \\ E.J.van.Leeuwen@ii.uib.no \\ 2 Department of Information and Computing Sciences, Utrecht University \\ P.O. Box 80.089, NL-3508 TB Utrecht, The Netherlands \\ j.vanleeuwen@cs.uu.nl
}

\begin{abstract}
Geometric intersection graphs are graphs determined by intersections of geometric objects. We study the complexity of visualizing the arrangements of objects that induce such graphs. We give a general framework for describing geometric intersection graphs, using arbitrary finite base sets of rationally given convex polygons and affine transformations. We prove that for every class of intersection graphs that fits the framework, the graphs in the class have a representation using polynomially many bits. Consequently, the recognition problem of these classes is in NP (and thus NP-complete). We also give an algorithm to find a drawing of the objects in the plane, if a graph class fits the framework.
\end{abstract}

\section{Introduction}

A geometric intersection graph is the intersection graph of a finite set of geometric objects. There is an edge between two vertices in the graph iff their corresponding objects intersect. The objects form a representation of the graph. Classes of geometric intersection graphs are obtained if one only allows objects similar to certain base objects specific for a class.

To visualize a geometric intersection graph, drawing a representation is more informative than just drawing the graph itself. Therefore we study the complexity of visualizing representations. We consider the following problems: do representations in polynomial space exist and if so, how can their drawings be found effectively.

Understanding Geometric Intersection Graphs. Geometric intersection graphs arise naturally in many areas. They are used e.g. in modeling wireless communication networks, where geometric objects model the transmission ranges of the different devices in the network. This has lead to the study of the well-known class of (unit) disk graphs and many other classes [9202223].

Current geometric intersection graph models use only homothetic copies, thus translations and scalings, of objects. Moreover, normally only a single base object is used. A broader notion of similarity and a larger variety of base objects may be desired in defining a class. Therefore we aim at a more general conceptual framework.

U. Brandes and S. Cornelsen (Eds.): GD 2010, LNCS 6502, pp. 377-388, 2011.

(C) Springer-Verlag Berlin Heidelberg 2011 
Definition 1. A signature is any 2-tuple $\mathcal{P}=\langle S, T\rangle$ with: (a) $S=\left\{o_{1}, \cdots, o_{m}\right\}$ is a finite nonempty base set of geometric objects in the plane, with each object in $S$ containing the origin, and (b) $T$ maps every object $o \in S$ to a finite set of similarity templates that determine how objects similar to o can be obtained.

Here a similarity template is any family of similarity transforms, e.g. a rotation over some angle, followed by a translation. More generally, a template $t$ will be any parametric family of bi-continuous functions $t\left(w_{1}, \ldots, w_{k}\right): \mathbb{R}^{2} \rightarrow \mathbb{R}^{2}$ which are shape-preserving in some sense, with the $w_{i}$ 's ranging over e.g. $\mathbb{R}_{+}$.

Definition 2. Given a signature $\mathcal{P}=\langle S, T\rangle$, a graph $G$ is called $\mathcal{P}$-intersection graph if it is the intersection graph of a finite set of objects $\mathcal{O}_{1}, \cdots, \mathcal{O}_{n}$, where every $\mathcal{O}_{i}(1 \leq i \leq n)$ is similar to an object $o \in S$, i.e. obtained using a transformation conforming to a similarity template in $T(o)$.

Problem Definitions. In order to visualize $\mathcal{P}$-intersection graphs, we must know the complexity of their representation. In particular, we want to know whether representations exist that require only polynomially many bits. Assume from now on that all objects we consider are fully specified, both for localizing and drawing them, by only finitely many parameters.

Definition 3. (i) $A \mathcal{P}$-intersection graph with $n$ vertices is said to be polynomially represented (using polynomial $p$ ), if it is the intersection graph of a finite set of objects $\mathcal{O}_{1}, \cdots, \mathcal{O}_{n}$, where every $\mathcal{O}_{i}(1 \leq i \leq n)$ is similar to an object in $S$ according to an allowed template, and has all its specifying parameters equal to rationals $\frac{a}{b}$ with $|a|,|b| \leq 2^{p(n)}$.

(ii) A class $\mathcal{C}$ of $\mathcal{P}$-intersection graphs is said to be polynomially represented if there is a polynomial $p=p(n)$ such that every graph in $\mathcal{C}$ is polynomially represented using $p$.

Given a graph, we like to determine whether it is a geometric intersection graph of some kind and be able to visualize it by its representation. This leads to the following problems.

\section{$\mathcal{P}$-Intersection Graph Recognition}

Given a graph $G$, decide whether $G$ is a $\mathcal{P}$-intersection graph.

\section{$\mathcal{P}$-Intersection Graph Construction (Visualisation)}

Given a graph $G$ that is known to be a $\mathcal{P}$-intersection graph, construct a representation of $G$ by objects in the plane according to signature $\mathcal{P}$.

We consider the complexity of both problems for $\mathcal{P}$-intersection graphs and whether or not such graphs have feasible, i.e. polynomial, representations.

Previous Work. The size of a representation and the complexity of the recognition problem have been studied for many classes of geometric intersection graphs. Some prominent results in this area are shown in Table 1. 
Table 1. Some classes of geometric intersection graphs. The first column indicates the graph class, the second column the objects used in representing the class, the third the complexity of the recognition problem, the fourth the size of a representation of the intersection graph (polynomial or exponential). The fifth gives references. Contributions of this paper are marked in italics. We use ${ }^{*}$ to refer to the current paper.

\begin{tabular}{|l|l|l|c|c|}
\hline Graph Class & Objects & Recognition & Repr. & Reference \\
\hline (unit) disk & disks & NP-h, $\in$ PSPACE & exp. & 1114919 \\
box (rectangle) & rectangles in $\mathbb{R}^{2}$ & NP-c & poly & {$[1318]$} \\
unit square & unit squares & NP-c & poly & {$[14]$} \\
square & squares & NP-h, $\in N P$ & poly & $*$ \\
max-tolerance & semi-squares & $\mathrm{NP}-\mathrm{h}, \in N P$ & poly & {$[10, *$} \\
polygon intersect. & homoth. conv. polygons & $\mathrm{NP}-\mathrm{h}$ & - & {$[1621]$} \\
polygon intersect. & rat. repr. conv. polygons & $\in N P$ & poly & $*$ \\
convex intersect. & convex sets $\subset \mathbb{R}^{2}$ & $\mathrm{NP}-\mathrm{h}, \in \mathrm{PSPACE}$ & exp. & {$[1221$} \\
\hline
\end{tabular}

Recognition can be nontrivial. For example, for disk graphs the problem is algorithmically decidable ([22]), known to be NP-hard [14] and in PSPACE [915], but it is open whether the problem actually is in NP. This holds even for the class of unit disk graphs.

The complexity or size of a representation poses an equally challenging problem. A first question is whether a class actually has a representation using only rational coordinates. This was shown e.g. for the intersection graphs of all socalled scalable objects 23 . If one only allows objects to touch but not to overlap (contact graphs), this is no longer guaranteed [3].

A second question is whether the rationals in these representations can be specified using polynomially many bits. For (unit) disk graphs, this question was answered negatively only recently [19.

Our Results. We apply our framework to define classes that use finite base sets of rationally given convex polygons and templates of rationally constrained affine transformations (see Section 21). We prove that for any such class, the intersection graphs in it have a polynomial representation, even in integers. This contrasts the known fact that intersection graphs of arbitrary convex polygons may require exponentially-sized representations in worst case [21].

We also settle, in a general way, the question left open by the recent NPhardness proof of the recognition problem for intersection graphs of homothetic copies of a single convex polygon [16, namely whether this problem is in NP. Our results immediately imply that this problem is indeed in NP, even for considerably larger classes of intersection graphs. Moreover, we give an algorithm to determine whether a given graph is an intersection graph within the above framework. The algorithm is constructive and returns a visualization of the arrangement of objects representing the given graph, if one exists.

The main result is presented in Section 4. (In Section 3 we show that it is irrelevant whether the objects we consider are open or closed.) By applying the same techniques, one can prove e.g. that max-tolerance graphs and contact 
graphs of homothetic convex polygons have polynomial representations, and that their recognition problems thus are in NP. Further applications are given in Section 5. More details are given in 25.

\section{$2 \mathcal{P}$-intersection Graphs}

$\mathcal{P}$-intersection graphs give a very general framework. We use it to consider $\mathcal{P}$ intersection graphs for signatures $\mathcal{P}=\langle S, T\rangle$, where $S$ is any finite nonempty base set of (closed and) rationally given convex polygons.

We also tune the choice of similarity templates. Similarity templates $t$ were described as parametric families of bi-continuous functions $t\left(w_{1}, \ldots, w_{k}\right): \mathbb{R}^{2} \rightarrow$ $\mathbb{R}^{2}$ which preserve shapes according to some notion of similarity. Templates should be smooth, which means that images of base objects under $t\left(w_{1}, \ldots, w_{k}\right)$ and $t\left(z_{1}, \ldots, z_{k}\right)$ must be 'almost equal' if $\left(w_{1}, \ldots, w_{k}\right)$ and $\left(z_{1}, \ldots, z_{k}\right)$ are.

Given convex base polygons, we restrict ourselves to linear similarity templates consisting of parameterized affine transformations over $\mathbb{Q}$ only.

Definition 4. A linear similarity template $t=t_{\alpha, \beta, \gamma, \delta}$ is a family of affine transformations of the form $x \rightarrow u+Q(v) x$, where: (i) $\alpha, \beta, \gamma, \delta$ are rationals such that $\alpha \delta-\beta \gamma \neq 0$, (ii) $u=\left(u_{1}, u_{2}\right)$ is any 2-dimensional vector, and (iii) $Q(v)=v\left(\begin{array}{ll}\alpha & \gamma \\ \beta & \delta\end{array}\right)$ is a $2 \times 2$ matrix with $v$ satisfying $v>0$.

Linear similarity templates have two parameters: $u$ (the translation vector) and $v$ (the scaling factor of the distortion matrix). Neither of them needs to be rational. The constraint $v>0$ keeps $Q(v)$ nonsingular and guarantees that all template mappings are topological isomorphisms. Linear similarity templates can be shown to be smooth. From now on, we only consider linear similarity templates.

Even though applying linear similarity transformations $x \rightarrow u+Q(v) x$ to objects $o$ amounts to applying regular homothetic transformations to objects $Q(o)$, the framework gives us the conceptual generality we want. Also, the framework allows us to vary templates while keeping the set of base polygons fixed.

An affine transformation $u+Q(v) x \in t$, where $t$ is any template assigned to a polygon in $S$ by $T$, is called a $\mathcal{P}$-transformation. Many familiar transformations (combined with scaling) are $\mathcal{P}$-transformations, aside from shifts and (skewed) scalings: horizontal shears, vertical shears, rotations, and reflexions, where for the latter the coefficients of the appropriate $Q$ must be rounded to keep them rational. $T$ may assign different sets of templates to different objects, without any dependency between them.

Definition 5. Given a signature $\mathcal{P}=\langle S, T\rangle$, object $\mathcal{O}$ is said to be similar to an object $o \in S$ if $\mathcal{O}$ can be obtained from o by applying an allowed $\mathcal{P}$-transformation to it.

One can show that the notion of similarity is well-founded, i.e. it is decidable, for any signature $\mathcal{P}=\langle S, T\rangle$ and convex polygon $\mathcal{O}$, whether $\mathcal{O}$ is similar to a polygon in $S$ under $\mathcal{P}$-transformation. Consider the (polynomial) representation of any $\mathcal{P}$-intersection graph and an arbitrary object $\mathcal{O}$ occurring in it. 
Lemma 1. Let $\mathcal{P}=\langle S, T\rangle$ be as above, and let $\mathcal{O}$ be similar to $o \in S$.

(i) If $\mathcal{O}$ is polynomially represented and $\mu>0$ is a polynomially represented rational, then the scaling of $\mathcal{O}$ by $\mu$ is also polynomially represented.

(ii) If $\mathcal{O}$ is polynomially represented and $\rho>0$ is a polynomially represented rational, then there is an enlargement of $\mathcal{O}$ by an additive margin $\delta$ with $0<$ $\delta<\rho$ that is again polynomially represented. This holds likewise for reductions.

Because the matrices $Q(v)$ in similarity templates are nonsingular, $\mathcal{P}$-transforms map convex polygons 1-1 onto convex polygons. Thus vertices and edges of the latter are images of the vertices and edges of the former, respectively. Recall that a convex polygon can be given by its defining inequalities.

Lemma 2. (i) Let $Q$ be a nonsingular $2 \times 2$ matrix, and o a plane convex polygon containing the origin. When $Q$ transforms o and $\operatorname{det} Q>0$, then defining inequalities are mapped to defining inequalities with preservation of the inequality sign. If $\operatorname{det} Q<0$, the inequality signs are reversed.

(ii) A P-transformation $\left(u_{1}, u_{2}\right)+Q(v) x$ with $Q(v)=v\left(\begin{array}{ll}\alpha & \gamma \\ \beta & \delta\end{array}\right)$ maps the line $a x+b y+c=0$ onto the line $(a \delta-b \beta) x+(b \alpha-a \gamma) y+(\alpha \delta-\beta \gamma) v c-(a \delta-$ $b \beta) u_{1}-(b \alpha-a \gamma) u_{2}=0$.

Lemma 2 enables one to determine exactly how the defining inequalities of a base polygon are transformed under a $\mathcal{P}$-transformation.

\section{Open Versus Closed Objects}

Let $\mathcal{P}=\langle S, T\rangle$ be a signature. What happens if we let $S$ consist of open convex polygons instead of closed ones? For disk graphs, it is known that taking open or closed disks does not change the class of graphs [24]. In [23], this was proved for the intersection graphs of all 'scalable' geometric objects. In the case of (unit) disk graphs, even polynomial representation is preserved.

We consider the case of $\mathcal{P}$-intersection graphs, emphasizing polynomial representation. We show in two steps that for $\mathcal{P}$-intersection graphs the closed and open cases are again equivalent. We use the following facts.

Lemma 3. Let $\mathcal{O}_{1}$ and $\mathcal{O}_{2}$ be two disjoint convex polygons in the plane, both having nonempty interior. The (shortest) distance between $\mathcal{O}_{1}$ and $\mathcal{O}_{2}$ is realized as the distance between a vertex of one polygon and an edge of the other.

Proof. This follows by a simple extension of the proof of Lemma 2.1 in [6].

Lemma 4. Let $a, b, c, v_{1}, v_{2}$ be rationals with their numerator and denominator bounded in absolute value by $q$ for some $q>0$. If the following fraction is $\neq 0$, then $\frac{\left|a v_{1}+b v_{2}+c\right|}{\sqrt{a^{2}+b^{2}}} \geq \frac{1}{2 q^{5}}$.

We first show one side of the equivalence. Let $\mathcal{P}=\langle S, T\rangle$. 
Lemma 5. Every polynomially represented $\mathcal{P}$-intersection graph using a non-empty base set of closed convex polygons can be obtained as a polynomially represented $\mathcal{P}$-intersection graph using a nonempty base set of open convex polygons.

Proof. Let $S=\left\{o_{1}, \cdots, o_{m}\right\}$ be the base set of closed convex polygons. Let $G$ be the intersection graph defined by the objects $\mathcal{O}_{1}, \cdots, \mathcal{O}_{n}$, where $\mathcal{O}_{i}(1 \leq i \leq n)$ is similar to $o_{s_{i}} \in S$ for some $s_{i} \in\{1, \ldots, m\}$ and obtained by applying an allowed affine transformation of $T\left(o_{s_{i}}\right)$ to $o_{s_{i}}$. Let $\mathcal{O}_{i}(1 \leq i \leq n)$ only have vertices with rational coordinates $\frac{a_{i}}{b_{i}}$ with $\left|a_{i}\right|,\left|b_{i}\right| \leq 2^{p(n)}$.

Let $\mathcal{P}^{\prime}=\left\langle S^{\prime}, T\right\rangle$ be the signature obtained from $\mathcal{P}$ in which every (closed) base polygon $o$ is replaced by its interior $o^{\circ}$. G can be viewed as the intersection graph of $\mathcal{O}_{1}^{\circ}, \cdots, \mathcal{O}_{n}^{\circ}$, provided no intersections of polygons are lost by restricting to the interiors. Intersections are lost precisely when there are (closed) polygons $\mathcal{O}_{i}$ and $\mathcal{O}_{j}$ that touch. We show that one can slightly enlarge the polygons $\mathcal{O}_{i}$ such that this does not occur, while preserving $G$ as the intersection graph.

Suppose one of the closed polygons, say $\mathcal{O}_{i}$, touches several other polygons $\mathcal{O}_{j}$. By enlarging $\mathcal{O}_{i}$ by a small but nonzero margin $\mu$, we can eliminate the touchings and let $\mathcal{O}_{i}$ overlap nontrivially with each $\mathcal{O}_{j}$. However, in enlarging it (and enlarging all other polygons for which this step is carried out) we must make sure that no spurious intersections with objects $\mathcal{O}_{r}$ disjoint from $\mathcal{O}_{i}$ are created. Suppose $\mathcal{O}_{i}$ and $\mathcal{O}_{r}$ are disjoint. By Lemma 3, the distance between them is realized by the distance between a vertex, say $v=\left(v_{1}, v_{2}\right)$ of one of them and an edge, say $a x+b y+c=0$ of the other. This distance is $\frac{\left|a v_{1}+b v_{2}+c\right|}{\sqrt{a^{2}+b^{2}}}$.

The numerators and denominators of $v_{1}, v_{2}$ are $\leq q=2^{p(n)}$. Also, $a x+b y+c=$ 0 connects two vertices of a polygon in the set, which have rational coordinates with numerators and denominators $\leq q$. It follows that $a, b, c$ are all rational, with numerators and denominators $\leq 4 q^{4}$. By Lemma 4 the distance is now at least $\frac{1}{d q^{20}}$ for some constant $d>0$. Hence, if we enlarge $\mathcal{O}_{i}$ by a nonzero margin of $\mu \leq \frac{1}{3 d q^{20}}$, then disjointness with every disjoint $\mathcal{O}_{r}$ is maintained (taking into account that the latter may also be enlarged by the same factor).

As $\mu$ is independent of the specific $\mathcal{O}_{i}$ chosen, the enlargement can be carried out simultaneously for all polygons. For every $\mathcal{O}_{i}$, it preserves all the intersections with other polygons, introduces no new ones, and has the effect that every polygon $\mathcal{O}_{i}$ that it touched, now overlaps nontrivially with it as well (and thus their interiors overlap). By Lemma 1 , enlarging every $\mathcal{O}_{i}$ by a nonzero margin at most $\mu$ can be achieved while preserving similarity and polynomial representation. Thus, $G$ is a $\mathcal{P}^{\prime}$-intersection graph and polynomially represented.

The converse of the lemma is proved in a similar way. We conclude:

Theorem 1. Every polynomially represented $\mathcal{P}$-intersection graph with a base set of closed convex polygons can be obtained as a polynomially represented $\mathcal{P}$-intersection graph with a base set of open convex polygons, and vice versa. 


\section{Representing $\mathcal{P}$-intersection Graphs}

Let $G$ be a $\mathcal{P}$-intersection graph and let some geometric realization of $G$ as $\mathcal{P}$ intersection graph be given. The realization of $G$ can be viewed as a feasible solution of a model, namely of a model that defines the exact pattern of intersections and nonintersections between the polygons. We will design a suitable LP model for this such that, if it has a feasible solution (which it has), it also has one that is polynomially represented, thus implying a geometric realization of $G$ with this property. A similar approach was used in 824]. We rely on the following fact, which seems folklore (cf. [10|15/25]).

Lemma 6. Two closed convex polygons in the plane are disjoint iff they can be separated by a line that precisely coincides with an edge of one of them.

Let $G$ be the $\mathcal{P}$-intersection graph of the convex polygons $\mathcal{O}_{1}, \cdots, \mathcal{O}_{n}$, where $\mathcal{O}_{i}$ $(1 \leq i \leq n)$ is similar to $o_{s_{i}} \in S$ (some $\left.s_{i} \in\{1, \ldots, m\}\right)$. Let $\mathcal{O}_{i}$ be the result of applying transformation $u_{i}+Q_{i} x=\left(\begin{array}{l}u_{i, 1} \\ u_{i, 2}\end{array}\right)+v_{i}\left(\begin{array}{ll}\alpha_{i} & \gamma_{i} \\ \beta_{i} & \delta_{i}\end{array}\right)\left(\begin{array}{l}x \\ y\end{array}\right)$ to $o_{s_{i}}$, with suitable $u_{i, 1}, u_{i, 2}$, and $v_{i}(1 \leq i \leq n)$, all conforming to a template $t=t_{i}$ applicable to $o_{s_{i}}$. Let $o_{s_{i}}(1 \leq i \leq n)$ have $k_{i}$ vertices and (thus) $k_{i}$ edges. All data related to $o_{s_{i}}$ (vertices, edges, defining inequalities) will be super-indexed by $(i)$.

\subsection{Helpful Inequalities}

Consider any two polygons $\mathcal{O}_{i}, \mathcal{O}_{j}$ and suppose we want to express that they are disjoint. By Lemma 6 there must be a defining inequality of (say) $\mathcal{O}_{i}$ such that all of $\mathcal{O}_{j}$ does not satisfy it. Which of $\mathcal{O}_{i}, \mathcal{O}_{j}$ to take and which defining inequality, follows from the given geometric realization of $G$. Say the polygon to take is indeed $\mathcal{O}_{i}$ and that the defining inequality to take is the one obtained by applying $t_{i}$ to the defining inequality $a^{(i)} x+b^{(i)} y+c^{(i)} \leq / \geq 0$ of $o_{s_{i}}$. Lemma 2 implies that this defining inequality of $\mathcal{O}_{i}$ can then be written as

$$
\begin{aligned}
& \left(a^{(i)} \delta_{i}-b^{(i)} \beta_{i}\right) x+\left(b^{(i)} \alpha_{i}-a^{(i)} \gamma_{i}\right) y+\left(\alpha_{i} \delta_{i}-\beta_{i} \gamma_{i}\right) v_{i} c^{(i)}-\left(a^{(i)} \delta_{i}-b^{(i)} \beta_{i}\right) u_{i, 1}- \\
& \left(b^{(i)} \alpha_{i}-a^{(i)} \gamma_{i}\right) u_{i, 2} \leq / \geq 0 .
\end{aligned}
$$

Each vertex of $\mathcal{O}_{j}$ is obtained from a vertex $\left(\begin{array}{c}d^{(j)} \\ e^{(j)}\end{array}\right)$ of $o_{s_{j}}$ using $u_{j}+Q_{j} x$, and can thus be written as $\left(\begin{array}{c}u_{j, 1}+\alpha_{j} d^{(j)} v_{j}+\gamma_{j} e^{(j)} v_{j} \\ u_{j, 2}+\beta_{j} d^{(j)} v_{j}+\delta_{j} e^{(j)} v_{j}\end{array}\right)$. To express that $\mathcal{O}_{j}$ is disjoint of $\mathcal{O}_{i}$ it now suffices to express that none of these $k_{j}$ vertices of $\mathcal{O}_{j}$ satisfy the defining inequality. This gives $k_{j}$ constraints of the form

$$
\begin{aligned}
& D I S J_{i, j}\left(u_{i, 1}, u_{i, 2}, v_{i}, u_{j, 1}, u_{j, 2}, v_{j}\right):= \\
& \left(a^{(i)} \delta_{i}-b^{(i)} \beta_{i}\right)\left(u_{j, 1}+\alpha_{i} d^{(j)} v_{j}+\gamma_{j} e^{(j)} v_{j}\right)+\left(b^{(i)} \alpha_{i}-a^{(i)} \gamma_{i}\right)\left(u_{j, 2}+\beta_{j} d^{(j)} v_{j}+\right. \\
& \left.\delta_{j} e^{(j)} v_{j}\right)+\left(\alpha_{i} \delta_{i}-\beta_{i} \gamma_{i}\right) v_{i} c^{(i)}-\left(a^{(i)} \delta_{i}-b^{(i)} \beta_{i}\right) u_{i, 1}-\left(b^{(i)} \alpha_{i}-a^{(i)} \gamma_{i}\right) u_{i, 2}>/<0
\end{aligned}
$$

one for each vertex of $\mathcal{O}_{j}$. We strengthen each inequality to " $\geq$ some positive margin" or " $\leq$ some negative margin" respectively (for real nonzero margins), by evaluating the inequalities in the given realization of $G$. The inequalities are homogeneous in $u_{i, 1}, u_{i, 2}, v_{i}, u_{j, 1}, u_{j, 2}, v_{j}$. Thus, multiplying all $u_{i, 1}, u_{i, 2}, v_{i}, u_{j, 1}$, $u_{j, 2}, v_{j}(1 \leq i, j \leq n)$ by a factor $\mu \geq 1$ large enough and rescaling the variables, the constraints still express the realization of $G$, but now with inequalities 
$D I S J_{i, j}\left(u_{i, 1}, u_{i, 2}, v_{i}, u_{j, 1}, u_{j, 2}, v_{j}\right) \leq-1 / \geq 1$ for $k_{j}$ nodes and relevant $1 \leq i, j \leq n$.

(We will in fact choose $\mu$ large enough such that a number of further goals w.r.t. the other constraints are achieved as well, as explained below.)

Next, $\mathcal{O}_{i}, \mathcal{O}_{j}$ overlap iff there is a point $\left(x_{i, j}, y_{i, j}\right)$ satisfying the defining inequalities of both $\mathcal{O}_{i}$ and $\mathcal{O}_{j}$. This leads to $k_{i}+k_{j}$ linear constraints

$$
\begin{aligned}
& I N_{i}\left(x_{i, j}, y_{i, j}, u_{i, 1}, u_{i, 2}, v_{i}\right) \leq / \geq 0, \\
& I N_{j}\left(x_{i, j}, y_{i, j}, u_{j, 1}, u_{j, 2}, v_{j}\right) \leq / \geq 0,
\end{aligned}
$$

one for each defining inequality of $\mathcal{O}_{i}$ and $\mathcal{O}_{j}$. The inequalities are homogeneous in $x_{i, j}, y_{i, j}, u_{i, 1}, u_{i, 2}, v_{i}, u_{j, 1}, u_{j, 2}, v_{j}$ and thus scale along with the scaling of the $D I S J$-inequalities.

Observe that the constraints " $v_{i}>0$ " may be replaced by " $v_{i} \geq$ some positive margin" in all cases as before, using the data from the given embedding. If we multiply all variables by a $\mu \geq 1$ large enough and rescale the variables accordingly, we can achieve that all constraints continue to express what we want, i.e. the resulting model still realizes $G$, but now we can also assume w.l.o.g. that $v_{i} \geq 1$ for $1 \leq i \leq n$.

Finally, note that the arrangement of polygons realizing $G$ can be shifted over any fixed vector we want. Thus, w.l.o.g. we may assume that $u_{i, 1}, u_{i, 2} \geq 0$ for every $1 \leq i \leq n$.

\subsection{Assembling the Model}

The model is complete when we define the situation (intersection or not) for every pair $\mathcal{O}_{i}, \mathcal{O}_{j}$. First include all constraints of the affine transformations $u_{i}+Q_{i} x=$ $\left(\begin{array}{l}u_{i, 1} \\ u_{i, 2}\end{array}\right)+v_{i}\left(\begin{array}{ll}\alpha_{i} & \gamma_{i} \\ \beta_{i} & \delta_{i}\end{array}\right)\left(\begin{array}{l}x \\ y\end{array}\right)$, following the templates $t_{i}$ that are used and taking the scalings into account: $u_{i, 1}, u_{i, 2} \geq 0$ and $v_{i} \geq 1$. (The condition $\alpha_{i} \delta_{i}-\beta_{i} \gamma_{i} \neq 0$ can be assumed for all templates in $T$.)

Next consider all $\frac{1}{2} n(n-1)$ pairs $\mathcal{O}_{i}, \mathcal{O}_{j}$ and express the model inequalities for each pair. For any pair $\mathcal{O}_{i}, \mathcal{O}_{j}$ we have $k_{i j}$ or $l_{i, j}$ inequalities respectively of the following form:

if $\mathcal{O}_{i}, \mathcal{O}_{j}$ must be disjoint:

$k_{i j} \leq \max \left\{k_{i}, k_{j}\right\}$ inequalities of type

$D I S J_{i, j}\left(u_{i, 1}, u_{i, 2}, v_{i}, u_{j, 1}, u_{j, 2}, v_{j}\right) \leq-1 / \geq 1$

if $\mathcal{O}_{i}, \mathcal{O}_{j}$ must intersect ( $m$ cases):

$l_{i j}=k_{i}+k_{j}$ inequalities of type

$I N_{i}\left(x_{i, j}, y_{i, j}, u_{i, 1}, u_{i, 2}, v_{i}\right) \leq / \geq 0$, resp

$I N_{j}\left(x_{i, j}, y_{i, j}, u_{j, 1}, u_{j, 2}, v_{j}\right) \leq / \geq 0$.

Bring the linear system into standard form with nonnegative slack variables $z_{i}, w_{i j 1}, \cdots, w_{i j k_{i j}}, z_{i j 1}, \cdots, z_{i j l_{i j}}$, turning inequality into equality constraints:

$v_{i}-z_{i}=1$,

if $\mathcal{O}_{i}, \mathcal{O}_{j}$ must be disjoint:

$k_{i j}$ inequalities $D I S J_{i, j}\left(u_{i, 1}, u_{i, 2}, v_{i}, u_{j, 1}, u_{j, 2}, v_{j}\right) \pm w_{i j r}=\mp 1\left(w_{i j r}\right.$ used in the $r$-th inequality), 
if $\mathcal{O}_{i}, \mathcal{O}_{j}$ must intersect:

$l_{i j}$ inequalities $I N_{i}\left(x_{i, j}, y_{i, j}, u_{i, 1}, u_{i, 2}, v_{i}\right) \pm z_{i j r}=0$ and

$I N_{j}\left(x_{i, j}, y_{i, j}, u_{j, 1}, u_{j, 2}, v_{j}\right) \pm z_{i j r}=0$ ( $z_{i j r}$ used in the $r$-th inequality),

now with the standard constraints: $u_{i, 1}, u_{i, 2}, v_{i}, x_{i, j}, y_{i, j}, z_{i} \geq 0(1 \leq i \leq n)$, $w_{i j 1}, \cdots, w_{i j k_{i j}} \geq 0(1 \leq i<j \leq n)$, and $z_{i j 1}, \cdots, z_{i j l_{i j}} \geq 0(1 \leq i<j \leq n)$. Note that all linear equations of the model have rational coefficients.

\subsection{Solving the Model}

Because $S$ is finite, there is a constant $k$ such that $k_{i} \leq k$ for every $1 \leq i \leq n$. As $T$ only has finitely many different templates, there is a constant $q \geq 1$ such that for every $1 \leq i \leq n$, the numerators and denominators of the (rational) coefficients of the defining inequalities of every $o_{s_{i}}$ and of the rationals $\alpha_{i}, \beta_{i}, \gamma_{i}, \delta_{i}$ are all $\leq 2^{q}$. Thus the coefficients in the linear inequalities $D I S J_{i, j}\left(u_{i, 1}, u_{i, 2}, v_{i}, u_{j, 1}, u_{j, 2}, v_{j}\right) \leq-1 / \geq 1$ and $I N_{i, j}\left(u_{i, 1}, u_{i, 2}, v_{i}, u_{j, 1}, u_{j, 2}, v_{j}\right)$ $\leq 0 / \geq 0$ are all rationals with numerators and denominators $\leq 2^{d q}$ for a small integer constant $d \geq 1$. The same bound holds in the standard form.

Let $N=n+\sum_{i j} k_{i j}+\sum_{i j} l_{i j} \leq n+\frac{1}{2} k n(n-1)$. The system of linear equalities can be written as: $\mathbf{A x}=\mathbf{b}$ with $\mathbf{x} \geq 0$, where

A is a $N$ by $N+3 n+2 m$ all-rational matrix, all entries $a$ of $\mathbf{A}$ have numerator and denominator $\leq 2^{d q}, N$ columns of $\mathbf{A}$ are unit vectors namely those corresponding to variables $z_{i}, z_{i j 1}, \cdots, z_{i j k_{i j}}, w_{i j 1}, \cdots, w_{i j l_{i j}}(1 \leq i \leq n$ and $1 \leq$ $i<j \leq n$ resp. $), \mathbf{x}=\left(\cdots, u_{i, 1}, u_{i, 2}, \cdots, v_{i}, \cdots, x_{i, j}, y_{i, j}, \cdots, z_{i}, \cdots, w_{i j 1}, \cdots\right.$, $\left.w_{i j k_{i j}}, \cdots, z_{i j 1}, \cdots, z_{i j l_{i j}}, \cdots\right)^{T}$, and $\mathbf{b}=(\cdots, 1, \cdots, \mp 1, \cdots, 0, \cdots)^{T}$, with all entries rational, in fact \pm 1 or 0 .

The term 'unit vector' is used to denote any column that has only one nonzero entry, with this entry being \pm 1 . Note that $\operatorname{rank}(\mathbf{A})=N=O\left(n^{2}\right)$.

Theorem 2. The LP model has an all-rational solution for $u_{i, 1}, u_{i, 2}, v_{i}$ with numerators and denominators bounded in absolute value by $2^{\mathcal{O}\left(n^{4}\right)}$.

Proof (Outline). Because $\mathbf{A x}=\mathbf{b}$ with $\mathbf{x} \geq 0$ has a feasible solution and $\operatorname{rank}(\mathbf{A})$ $=N$, it has a basic feasible solution with (at least) $3 n+2 m$ of the coordinates of $\mathbf{x}$ equal to 0 , whereas the $N$-by- $N$ submatrix $\mathbf{A}^{\prime}$ consisting of the columns corresponding to the other coordinates is invertible and satisfies $\mathbf{A}^{\prime} \mathbf{x}^{\prime}=\mathbf{b}$ (with $\mathbf{x}^{\prime} \geq 0$ ), where $\mathbf{x}^{\prime}$ is the subvector of $\mathbf{x}$ consisting of these other coordinates. Hence, by Cramer's rule, it follows that $\left(\mathbf{x}^{\prime}\right)_{i}=\frac{\operatorname{det} \mathbf{A}_{\mathbf{i}}^{\prime}}{\operatorname{det} \mathbf{A}^{\prime}}$, where $\mathbf{A}_{\mathbf{i}}^{\prime}$ is the matrix formed by replacing the $i$-th column of $\mathbf{A}^{\prime}$ by $\mathbf{b}$. Observe that, because $\mathbf{A}^{\prime}$ and $\mathbf{A}_{\mathbf{i}}^{\prime}$ are rational matrices, their determinants are rational as well.

The nonzero entries of $\mathbf{A}^{\prime}$ are all of the form $\frac{f}{h}$ with $f, h$ integer and $|f|,|h| \leq$ $2^{d q}$. Let $H_{i}$ be the product of all $|h|$-values that occur as denominators in the $i$-th column and let $H=\prod_{1}^{N} H_{i}$, thus $H \leq 2^{d q \cdot N \cdot N}$. Then $\operatorname{det} \mathbf{A}^{\prime}=\frac{1}{H} \operatorname{det} \mathbf{A}^{\prime \prime}$, where $\mathbf{A}^{\prime \prime}$ is obtained from $\mathbf{A}^{\prime}$ by multiplying the elements in the first column by $H_{1}$, the elements of the second column by $H_{2}$, etc. Using Hadamard's inequality 
for matrices, $F=\left|\operatorname{det} \mathbf{A}^{\prime \prime}\right| \leq\left(\sqrt{N 2^{2 d q N}}\right)^{N} \leq N^{\frac{1}{2} N} 2^{d q N^{2}} \leq 2^{2 d q N^{2}}$. This shows that $\operatorname{det} \mathbf{A}^{\prime}=\frac{F}{H}$ with $F, H$ integers with $|F|,|H| \leq 2^{g n^{4}}$ for some constant $g$. The same bound holds for the other determinants.

Theorem 2 gives a sufficient bound and easily leads to the following, main result.

Theorem 3. Let $G$ be a $\mathcal{P}$-intersection graph. Then $G$ has a polynomial representation, even fully in integers.

Corollary 1. The recognition problem for every class of $\mathcal{P}$-intersection graphs is in $N P$.

Although the above arguments seem to rely on being given some realization of the $\mathcal{P}$-intersection graph, this is in fact not necessary. It suffices to know for each vertex of $G$ which base polygon of $S$ and which transformations of $T$ to use, and for any nonadjacent pair of vertices which defining inequality of the objects representing these vertices to use to express disjointness. Quantifying over this information suffices to find a realization of $\mathcal{P}$.

Corollary 2. The construction (i.e. 'drawing') problem of any class of $\mathcal{P}$-intersection graphs can be solved algorithmically, in exponential time.

\section{Applications}

The notions of signatures and $\mathcal{P}$-intersection graphs are very useful in modeling classes of intersection graphs, particularly when combined with the generic theorems presented above. We list a few applications.

Square Intersection Graphs: It is known that unit square graphs have polynomial size representations [4. We can now extend this to square intersection graphs. Recall that NP-hardness of the recognition problem of unit square intersection graphs was proved in [1. For general square graphs, NP-hardness follows from the recent results in [16].

Theorem 4. Square intersection graphs have polynomial-size integer representations. Their recognition problem is in NP (and thus NP-complete).

Proof. Define signature $\mathcal{P}=\langle S, T\rangle$ with $S$ consisting of a unit square around the origin, and $T$ consisting of the template $t: u+v\left(\begin{array}{ll}1 & 0 \\ 0 & 1\end{array}\right)$. Square intersection graphs are precisely the $\mathcal{P}$-intersection graphs for this signature $\mathcal{P}$. Now apply Theorem 3 and Corollary 1.

Polynomial representation for unit square graphs is easily shown directly [4, but also follows from our arguments, by adding equations $v_{i}=v_{j}$ to the model.

Max-tolerance Graphs: Kaufmann et al. [10] showed that the max-tolerance graphs are precisely the intersection graphs of so-called semi-squares. (A semisquare is 'a square with one half cut off along the bottom-right to top-left diagonal'.) In the same way as in the previous example, one can show that semi-square 
intersection graphs, thus max-tolerance graphs, have polynomial-size integer representations and an NP-recognition problem.

As Kaufmann et al. [10] proved the recognition problem of max-tolerance graphs to be NP-hard, it now follows that this problem is in fact NP-complete.

Intersection Graphs of Homothetic Polygons: Kratochvíl and Pergel [16] initiated a general study of the intersection graphs that can be formed using homothetic copies of a single convex polygon $P$, or $P_{\text {hom-intersection }}$ graphs. (We assume that $P$ is always finitely given, in rational coordinates.) They show that the recognition problem for $P_{h o m}$-intersection graphs is NP-hard. We can strengthen this as follows.

Theorem 5. Phom-intersection graphs have polynomial-size integer representations. Their recognition problem is in NP (and thus NP-complete).

Proof. Define $\mathcal{P}=\langle S, T\rangle$ with $S=\{P\}$ and $T$ assigning the homothetic transformations to $P$. $P_{h o m}$-intersection graphs are precisely the $\mathcal{P}$-intersection graphs. The result follows.

In [16, Kratochvíl and Pergel also define $P_{h o m}$-contact graphs, where intersections are restricted to being contacts only. They pose as an open problem to determine the complexity of recognizing $P_{h o m}$-contact graphs. By modifying the LP model, one can show by the same technique as developed in Section 4 that $P_{h o m}$-contact graphs have polynomial-size integer representations. Thus the recognition problem for $P_{h o m}$-contact graphs is in $N P$. It remains open whether this problem is NP-complete.

Note added in proof: In recent work jointly with Tobias Müller (CWI, Amsterdam), tight upper- and lowerbounds have been obtained on the number of bits needed for representing convex polygon intersection graphs.

\section{References}

1. H.: Breu, Algorithmic aspects of constrained unit disk graphs, PhD Thesis, The University of British Columbia, Vancouver (1996)

2. Breu, H., Kirkpatrick, D.G.: Unit disk graph recognition is NP-hard. Computational Geometry 9, 3-24 (1998)

3. Brightwell, G.R., Scheinerman, E.R.: Representations of planar graphs. SIAM Journal of Discrete Mathematics 6(2), 214-229 (1993)

4. Czyzowicz, J., Kranakis, E., Krizanc, D., Urrutia, J.: Discrete realizations of contact and intersection graphs. Int. J. Pure and Applied Mathematics 13(4), 429-442 (2004)

5. Deng, X., Hell, P., Huang, J.: Linear time representation of proper circular arc graphs and proper interval graphs. SIAM Journal of Computing 25, 390-403 (1996)

6. Edelsbrunner, H.: Computing the extreme distances between two convex polygons. J. of Algorithms 6, 213-224 (1985)

7. Golumbic, M.C., Trenk, A.N.: Tolerance graphs. Cambridge University Press, Cambridge (2004) 
8. Hayward, R.B., Shamir, R.: A note on tolerance graph recognition. Discrete Applied Mathematics 143, 307-311 (2004)

9. Hliněný, P., Kratochvíl, J.: Representing graphs by disks and balls (A survey of recognition-complexity results). Discrete Mathematics 229, 101-124 (2001)

10. Kaufmann, M., Kratochvíl, J., Lehmann, K.A., Subramanian, A.R.: Max-tolerance graphs as intersection graphs: cliques, cycles, and recognition. In: Proc. 17th Ann. ACM-SIAM Symp. on Discrete Algorithms (SODA 2006), pp. 832-841 (2006)

11. Kozyrev, V.P., Yushmanov, S.V.: Representations of graphs and networks (codings, layouts and embeddings). Journal of Soviet Mathematics 61(3), 2152-2194 (1992)

12. Kratochvíl, J., Matoušek, J.: NP-hardness results for intersection graphs. Commentationes Mathematicae Universitatis Carolinae 30(4), 761-773 (1989)

13. Kratochvíl, J.: A special planar satisfiability problem and a consequence of its NP-completeness. Discrete Applied Mathematics 52(3), 233-252 (1994)

14. Kratochvíl, J.: Intersection graphs of noncrossing arc-connected sets in the plane. In: North, S.C. (ed.) GD 1996. LNCS, vol. 1190, pp. 257-270. Springer, Heidelberg (1997)

15. Kratochvíl, J.: Geometric representations of graphs, Graduate Course, notes, Universitat Politècnica de Catalunya, Barcelona (April 2005), http://www.aco.gatech.edu/conference/archive/acokratochvil.ppt

16. Kratochvíl, J., Pergel, M.: Intersection graphs of homothetic polygons. In: Electronic Notes in Discrete Mathematics, vol. 31, pp. 277-280 (2008), http://www . canalc2.tv/video.asp?idvideo $=7571$

17. Lin, M.C., Szwarcfiter, J.L.: Unit circular-arc graph representations and feasible circulations. SIAM J. Discrete Mathematics 22(1), 409-423 (2008)

18. Lingas, A., Wahlen, M.: A note on maximum independent set and related problems on box graphs. Inf. Proc. Letters 93, 169-171 (2005)

19. McDiarmid, C., Müller, T.: The number of bits needed to represent a unit disk graph. In: Thilikos, D.M. (ed.) WG 2010. LNCS, vol. 6410, pp. 315-323. Springer, Heidelberg (2010)

20. McKee, T.A., McMorris, F.R.: Topics in intersection graph theory. SIAM Monographs on Discrete Mathematics and Applications, vol. 2, SIAM, Philadelphia (1999)

21. Pergel, M.: Special graph classes and algorithms on them, PhD Thesis, Dept. of Applied Mathematics, Charles University, Prague (2008)

22. Spinrad, J.R.: Efficient graph representations. In: Field Institute Monographs, vol. 19, American Mathematical Society, Providence (2003)

23. van Leeuwen, E.J.: Optimization and approximation on systems of geometric objects, PhD thesis, University of Amsterdam (2009)

24. van Leeuwen, E.J., van Leeuwen, J.: On the representation of disk graphs, Techn. Report UU-CS-2006-037, Dept. of Information and Computing Sciences, Utrecht University (2006)

25. van Leeuwen, E.J., van Leeuwen, J.: Convex polygon intersection graphs, Techn. Report, Dept. of Information and Computing Sciences, Utrecht University (to appear, 2010) 